\title{
A Stator Voltage Oriented PI Controller For The Doubly-Fed Induction Machine
}

\author{
Carles Batlle, Arnau Dòria-Cerezo and Romeo Ortega
}

\begin{abstract}
In this paper we propose a new control scheme for the doubly-fed induction machine (DFIM) that offers significant advantages, and is considerably simpler, than the classical vector control method. In contrast with the latter, where the DFIM is represented in a stator flux-oriented frame, we propose here a model with orientation of the stator voltage. This allows for an easy decomposition of the active and reactive powers on the stator side and their regulation-acting on the rotor voltage-via stator current control. Our main contribution is the proof that a linear PI control around the stator currents ensures global stability for a feedback linearized DFIM, provided the gains are suitably selected. The feedback linearization stage requires only measurement of the rotor and stator currents, hence is easily implementable. Furthermore, to improve the robustness, an adaptive version that estimates the rotor resistance is proposed. Tuning rules for the PI gains are also provided. Finally, an outer loop control for the mechanical speed is introduced. The complete control system is tested both in simulations and experiments, showing good transient performance and robustness properties.
\end{abstract}

\section{INTRODUCTION}

Doubly-fed induction machines (DFIM) have become very popular for renewable energy applications lately. They have been proposed in the literature, among other applications, for wind-turbine generators [10], hybrid engines [6] or high performance storage systems [3]. The attractiveness of the DFIM stems primarily from its ability to handle large speed variations around the synchronous speed (see [11] for an extended literature survey and discussion). Another advantage is that the power electronic equipment to control the machine only has to handle a fraction (maximum $20-30 \%$ ) of the total power, reducing the losses (and the cost) of the power electronic converter.

Most DFIM controllers proposed in the literature are based on vector control and decoupling [9], see examples in [10],

C.B. and A.D.C. were partially supported by the CICYT project DPI200406871-CO2-02. The work of R.O. was partially funded by the European Network of Excellence HyCON.

C. Batlle is with the Department of Applied Mathematics IV and Institute of Industrial and Control Engineering, Universitat Politècnica de Catalunya, 08800 Vilanova i la Geltrú, Spain carles . batl le@upc.edu

A. Dòria-Cerezo is with the Department of Electrical Engineering and Institute of Industrial and Control Engineering, Universitat Politècnica de Catalunya, 08800 Vilanova i la Geltrú, Spain arnau.doria@upc.edu

R. Ortega is with Laboratoire des Signaux et Systèmes, Supélec, Gif-surYvette, 91192, France romeo.ortega@lss.supelec.fr
[13]. This methodology is based on the description of the electrical part of the DFIM in a new reference frame (usually the stator flux), which allows the decoupling of the active and reactive power of the stator side and their independent control through the rotor currents. To achieve the stator flux orientation the flux angle must be computed and several complicated (and extremely fragile) rotation operations implemented. Other control schemes with rigorous stability and robustness analysis reported in the literature are the output feedback algorithm presented in [11], and the passivitybased controllers proposed in [3], [5].

This paper presents a new control algorithm for the DFIM that offers significant advantages, and is considerably simpler, than the previous control methods. In contrast with vector control, where the DFIM is represented in a stator flux-oriented frame, we propose here a model with orientation of the stator voltage. This allows for an easy decomposition of the active and reactive powers on the stator side and their regulation-acting on the rotor voltage-via stator current control. Our main contribution is the proof that a linear PI control around the stator currents ensures global stability for a feedback linearized DFIM-provided the gains are suitably selected. The feedback linearization stage requires only measurement of the rotor and stator currents, hence is easily implementable. Furthermore, to improve the robustness, an adaptive version that estimates the rotor resistance is proposed. Tuning rules for the PI gains are also provided, in particular, we prove that, if the integral gain is small, the proportional gain can take arbitrarily large values. Also, we prove the existence of large (open) regions in the controller parameter plane where stability is preserved. Finally, as done also in vector control, an outer loop control for the mechanical speed is introduced. The complete control system is tested both in simulations and in experiments, showing good transient performance and robustness properties.

\section{Model of the Doubly-Fed Induction Machine}

We start from the three phase dynamical equations of a DFIM, and assume that the machine is symmetric (all windings are equal), the stator-rotor cross inductances are smooth, sinusoidal functions of the rotor angle with just the fundamental term [8], and that the three phase system is 
equilibrated. These assumptions allow the use of transformations, which greatly simplifies the control problem. The transformations (also known as Blondel-Parks transformations) are widely used in the study of power systems [8]. This mathematical transformation is used to decouple one of the (balanced) phases, to refer all variables to a common reference frame, and to obtain constitutive laws (stator-rotor cross inductances) independent of the relative angle between rotor and stator.

Similarly to [3], in this paper we propose a transformation to a synchronous frame rotating at the frequency of the stator voltage of the DFIM, which is assumed constant. This yields

$$
\begin{aligned}
\dot{\lambda}_{s}= & -\left(\omega_{s} L_{s} J_{2}+R_{s} I_{2}\right) i_{s}-\omega_{s} L_{s r} J_{2} i_{r}+v_{s} \\
\dot{\lambda}_{r}= & -\left(\omega_{s}-\omega\right) L_{s r} J_{2} i_{s} \\
& -\left[\left(\omega_{s}-\omega\right) L_{r} J_{2}+R_{r} I_{2}\right] i_{r}+v_{r} \\
J \dot{\omega}= & L_{s r} i_{s}^{\top} J_{2} i_{r}-B_{r} \omega-\tau_{L}
\end{aligned}
$$

where $\lambda_{s}, \lambda_{r} \in \mathbb{R}^{2}$ are the stator and rotor fluxes, $i_{s}, i_{r} \in \mathbb{R}^{2}$ are the stator and rotor currents, $v_{s}=\operatorname{col}\left(V_{s}, 0\right) \in \mathbb{R}^{2}$, with $V_{s}$ the amplitude of the three-phase stator voltage, is the stator voltage, the rotor voltage $v_{r} \in \mathbb{R}^{2}$ is the control input, $\omega$ is the mechanical speed, and $\omega_{s}$ is the stator frequency. $R_{s}$, $R_{r}$ are the stator and rotor resistances, $L_{s}, L_{r}$ and $L_{s r}$ are the stator, rotor and self-inductances, with $L_{s} L_{r}>L_{s r}^{2}, J$ is the inertia, $B_{r}$ is the friction coefficient, $\tau_{L}$ is an external constant torque, and we defined the matrices

$$
J_{2}=\left[\begin{array}{cc}
0 & -1 \\
1 & 0
\end{array}\right] \quad I_{2}=\left[\begin{array}{ll}
1 & 0 \\
0 & 1
\end{array}\right] .
$$

Linking fluxes, $\lambda=\operatorname{col}\left(\lambda_{s}, \lambda_{r}\right)$, and currents, $i=\operatorname{col}\left(i_{s}, i_{r}\right)$, are related by $\lambda=L i$, where

$$
L=\left[\begin{array}{cc}
L_{s} I_{2} & L_{s r} I_{2} \\
L_{s r} I_{2} & L_{r} I_{2}
\end{array}\right]
$$

Following standard convention we partition all electrical (two-dimensional vector) signals into their, so-called, $d$ and $q$ components. For instance, the stator current is decomposed as $i_{s}=\operatorname{col}\left(i_{s d}, i_{s q}\right)$. The use of the synchronous frame allows us to express the stator active and reactive powers in terms of $i_{s d}$ and $i_{s q}$, respectively. In particular, assigning a desired value, $i_{s q}^{*}$, allows to compensate the power factor of the stator side of the machine, while $i_{s d}^{*}$ can be used to control the active power (delivered or consumed) by the DFIM. In a drive application, we can fix $i_{s d}^{*}$ as a desired value to achieve the target speed. In this paper we concentrate only on the problem of robust regulation of $i_{s}$ to its desired value and refer the interested reader to [3] for further details on the power flow control policy and the determination of the equilibria.

\section{Overall Control Scheme}

The proposed control scheme is presented in Fig. 1, where the current control block assures stability of the electrical subsystem and an outer-loop control is added for speed regulation. As indicated above, in this paper we concentrate on current control and will prove that, after a basic feedback linearization stage, the current can be globally regulated with a PI around the stator currents with some suitably selected gains.

The transformation of the three phase (stator and rotor) currents to the synchronous-reference (aligned to the stator voltages) is achieved with the rotation matrices

$$
K(\theta, \delta)=\left[\begin{array}{cc}
e^{J_{2} \delta} & O_{2} \\
O_{2} & e^{J_{2}(\delta-\theta)}
\end{array}\right],
$$

where $\delta$ is an arbitrary function of time that we select as $\dot{\delta}=$ $\omega_{s}$. Notice that this part of the scheme is easier to implement than vector control, which requires stator flux estimation.

\section{Current Controller}

The proposed controller consists of a feedback linearization stage

$$
v_{r}=\left(\omega_{s}-\omega\right) L_{s r} J_{2} i_{s}+\left[\left(\omega_{s}-\omega\right) L_{r} J_{2}+R_{r} I_{2}\right] i_{r}+u
$$

and a PI action

$$
u=-k_{P} J_{2} \tilde{i}_{s}+k_{I} J_{2} \int \tilde{i}_{s} d t
$$

with the scalar proportional and integral gains $k_{P}>0, k_{I} \geq$ 0 , respectively, and we defined the error terms $(\tilde{\cdot})=(\cdot)-(\cdot)^{*}$, where $(\cdot)^{*}$ is the constant desired value.

We attract the readers attention to the following important remarks:

R1. The first two terms in (4) exactly cancel the terms in (2), feedback linearizing the system and transforming the rotor equation into $\dot{\lambda}_{r}=u$. To improve the robustness of this stage we propose in Section $\mathrm{V}$ an adaptive implementation that estimates the highly uncertain rotor resistance.

R2. Due to the feedback linearization the overall system consists of a cascade of the electrical and the mechanical sub-systems. As the latter, (3), is a simple stable linear system, convergence to the equilibria of the electrical sub-system will imply stability of the complete dynamics.

R3. In contrast to standard practice, we have defined the PI, (5), with the skew-symmetric matrix $J_{2}$. Notice also the selection of the signs. These two features will be critical for the stability analysis. ${ }^{1}$

\footnotetext{
${ }^{1}$ As explained in [7] this controller was obtained applying passivity-based nonlinear control techniques, but here we restrict ourselves to its analysis.
} 


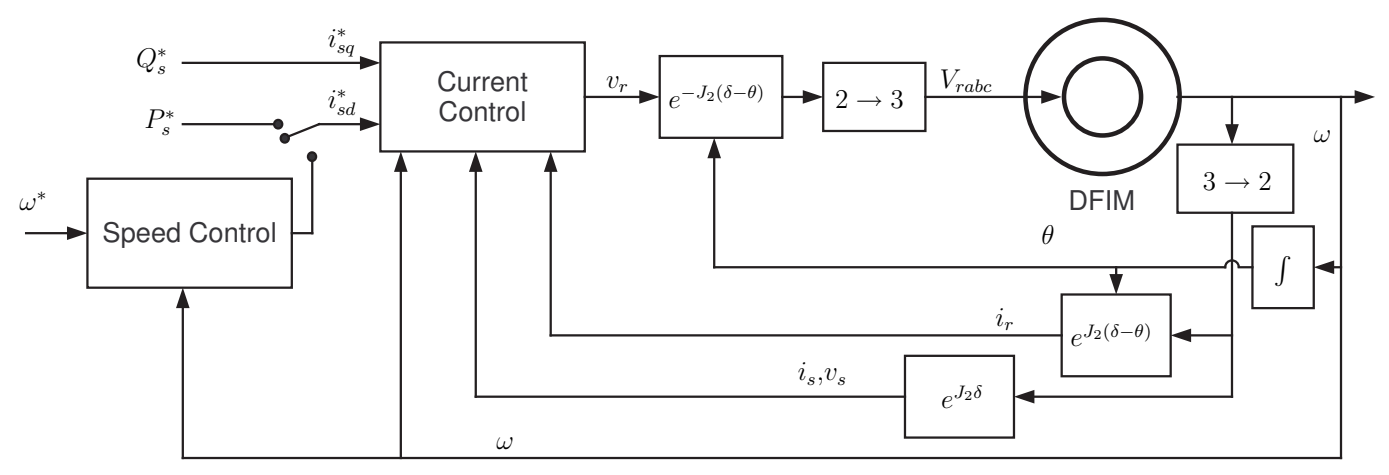

Fig. 1. Control scheme for a DFIM.

To carry out the stability analysis, we find convenient to express the closed-loop system in an alternative form. Replacing (4) and (5) in (1), (2), and using the definition of equilibria, we can write the closed-loop system in error coordinates as

$$
\begin{aligned}
& \dot{\tilde{\lambda}}_{s}=-\left(\omega_{s} L_{s} J_{2}+R_{s} I_{2}\right) \tilde{i}_{s}-\omega_{s} L_{s r} J_{2} \tilde{i}_{r} \\
& \dot{\tilde{\lambda}}_{r}=-k_{P} J_{2} \tilde{i}_{s}+k_{I} J_{2} \int \tilde{i}_{s} d t .
\end{aligned}
$$

Using the relation between fluxes and currents we get

$$
\dot{\tilde{\lambda}}_{s}=L_{s} \dot{\tilde{i}}_{s}+\frac{L_{s r}}{L_{r}}\left(\dot{\tilde{\lambda}}_{r}-L_{s r} \dot{\tilde{i}}_{s}\right) .
$$

Similarly $\tilde{i}_{r}=\frac{1}{L_{r}}\left(\tilde{\lambda}_{r}-L_{s r} \tilde{i}_{s}\right)$. Replacing the last two equations in (6), differentiating and using (7) we can write the electrical dynamics in the equivalent form

$$
D(p) \tilde{i}_{s}=0,
$$

with the polynomial matrix, in the derivative operator $p=\frac{d}{d t}$,

$$
D(p)=p^{3} I_{2}+\left(c_{1} I_{2}+c_{2} J_{2}\right) p^{2}+\left(c_{3} I_{2}+c_{4} J_{2}\right) p+c_{5} I_{2},
$$

and the parameters

$$
\begin{gathered}
c_{1}=\frac{R_{s} L_{r}}{\mu}, \quad c_{2}=\omega_{s}-\frac{L_{s r}}{\mu} k_{P}, \quad c_{3}=\frac{\omega_{s} L_{s r}}{\mu} k_{P}, \\
c_{4}=-\frac{L_{s r}}{\mu} k_{I}, \quad c_{5}=\frac{\omega_{s} L_{s r}}{\mu} k_{I},
\end{gathered}
$$

where $\mu=L_{s} L_{r}-L_{s r}^{2}>0$.

Equation (8) describes, of course, a linear system of order six whose stability is determined by the characteristic polynomial $\operatorname{det} D(s)$, with $s \in \mathbb{C}$ the Laplace transform variable. Although the study of this (sixth-order) polynomial can be carried out with classical tools, e.g., Routh-Hurwitz criterion, this procedure yields complex parameter relations that complicate the choice of the PI gains. On the other hand, we show now that the particular structure chosen for the PI, see R3 above, permits very simple analysis and tuning rules and ensures some interesting robustness properties. These results are contained in the following proposition, whose proof is given in [4].
Proposition 1: Consider the DFIM system (1)-(3) in closed-loop with the control (4) and (5).

P1. If $k_{I}=0$, for all $k_{P}>0$, the electrical coordinates converge to their desired values, while the speed is bounded and also converges to a constant value.

P2. There exists $k_{I}^{M}>0$ such that, for all $k_{I} \in\left(0, k_{I}^{M}\right]$ and all $k_{P}>0$, the electrical coordinates converge to their desired values, while the speed is bounded and also converges to a constant value.

Let us briefly explain the motivation behind the proposed PI (5). Towards this end, we attract the readers attention to the coefficients $c_{2}$ and $c_{3}$, that depend on $k_{P}$. Due to the particular choice of the proportional gain matrix we have that $c_{3}>0$. On the other hand, even though $c_{2}$ may become negative (for large values of $k_{P}$ ), this coefficient multiplies the skew-symmetric matrix $J_{2}$. To understand how this influences the stability let us consider first the case $k_{I}=0$. The dynamics of the system is then described by $D_{0}(p) \tilde{i}_{s}=0$, with

$$
D_{0}(p)=p^{2} I_{2}+\left(c_{1} I_{2}+c_{2} J_{2}\right) p+c_{3} I_{2} .
$$

It turns out that this system is asymptotically stable for all $k_{P}>0$. Indeed, consider a Lyapunov function candidate

$$
V\left(\tilde{i}_{s}, \dot{\vec{i}}_{s}\right)=\frac{1}{2}\left|\dot{\tilde{i}}_{s}\right|^{2}+\frac{c_{3}}{2}\left|\tilde{i}_{s}\right|^{2} \geq 0,
$$

with $|\cdot|$ the Euclidean norm and $c_{3}>0$. Taking the derivative of $V$ we get

$$
\dot{V}=-\left.c_{1} \dot{\tilde{\mid}}_{s}\right|^{2} \leq 0,
$$

which proves the claim. Observe that, since $c_{2}$ disappears in the computation of the derivative, its sign may be positive or negative without affecting the conclusion. This nice stability property is lost if the proportional gain matrix is not skewsymmetric.

A similar argument can be used to justify the choice of the integral gain matrix, as follows. The characteristic equation of the closed-loop system has the following form

$$
\operatorname{det} D(s)=s^{6}+a s^{5}+b s^{4}+c s^{3}+d s^{2}+e s+f,
$$


where

$$
\begin{aligned}
a & =\frac{1}{\mu} 2 L_{r} R_{s} \\
b & =\frac{1}{\mu^{2}}\left(\omega_{s}^{2} \mu^{2}+L_{s r}^{2} k_{P}^{2}+L_{r}^{2} R_{s}^{2}\right) \\
c & =\frac{1}{\mu^{2}} 2 L_{s r} k_{P}\left(R_{s} L_{r} \omega_{s}+L_{s r} k_{I}\right)=c_{0}+k_{I} c_{11} \\
d & =\frac{1}{\mu^{2}} L_{s r}\left(\omega_{s}^{2} L_{s r} k_{P}^{2}+L_{s r} k_{I}^{2}+2 L_{r} \omega_{s} R_{s} k_{I}\right) \\
& =d_{0}+k_{I} d_{1}+k_{I}^{2} d_{2} \\
e & =\frac{1}{\mu^{2}} 2 L_{s r}^{2} \omega_{s}^{2} k_{P} k_{I}=k_{I} e_{1} \\
f & =\frac{1}{\mu^{2}} L_{s r}^{2} \omega_{s}^{2} k_{I}^{2}=k_{I}^{2} f_{2},
\end{aligned}
$$

and we have factored the gain $k_{I}$. Thus,

$$
\begin{aligned}
\operatorname{det} D(s)= & s^{6}+a s^{5}+b s^{4}+c_{0} s^{3}+d_{0} s^{2} \\
& +k_{I}\left(c_{11} s^{3}+d_{1} s^{2}+e_{1} s\right)+k_{I}^{2}\left(d_{2} s^{2}+f_{2}\right) .
\end{aligned}
$$

For small $k_{I}$ the quadratic term can be disregarded and we can analyze the reduced polynomial $\beta(s) s+k_{I} \alpha(s)=0$, where

$$
\begin{aligned}
& \beta(s)=s^{4}+a s^{3}+b s^{2}+c_{0} s+d_{0} \\
& \alpha(s)=c_{11} s^{2}+d_{1} s+e_{1} .
\end{aligned}
$$

Note that $\beta(s)$ is the characteristic polynomial of the system with $k_{I}=0$, therefore its roots are always on the open lefthalf plane. On the other hand, the roots of $\alpha(s)$, given by,

$$
s_{1}, s_{2}=-\frac{d_{1}}{2 c_{11}} \pm \sqrt{\left(\frac{d_{1}}{2 c_{11}}\right)^{2}-\frac{e_{1}}{c_{11}}}
$$

have negative real part for $e_{1}, c_{11}>0$, which is true in our case. This analysis, combined with a continuity argument, provides a proof of claim P2 in Proposition 1.

Before closing this section we make the following remark. The result of Proposition 1 concerns stability of the closedloop system for any $k_{P}$ (even arbitrarily large) and for $k_{I}$ small enough. In fact, an asymptotic analysis of the Routh-Hurwitz conditions for the characteristic polynomial, $\operatorname{det} D(s)$, shows that there is an unbounded region in the first quadrant, below the line $k_{I}=\frac{L_{r} R_{s}}{\mu} k_{P}-\frac{L_{r} R_{s}}{L_{s r}} \omega_{s}$, where the closed-loop system is stable. Fig. 2 shows an sketch of the region of stability in the plane $\left(k_{P}, k_{I}\right)$ space.

\section{AdAPTIVE FEEDBACK LinEARIZATION}

As seen from (4) the feedback linearization term requires the exact knowledge of $R_{r}$, which is in general an uncertain parameter. To robustify the scheme we propose an adaptive implementation where we estimate this parameter. Unfortunately, the classical adaptation scheme depends, in a complicated way, on the parameters of the DFIM that need to be exactly know. To overcome this important practical shortcoming and obtain a simple robust adaptation law we

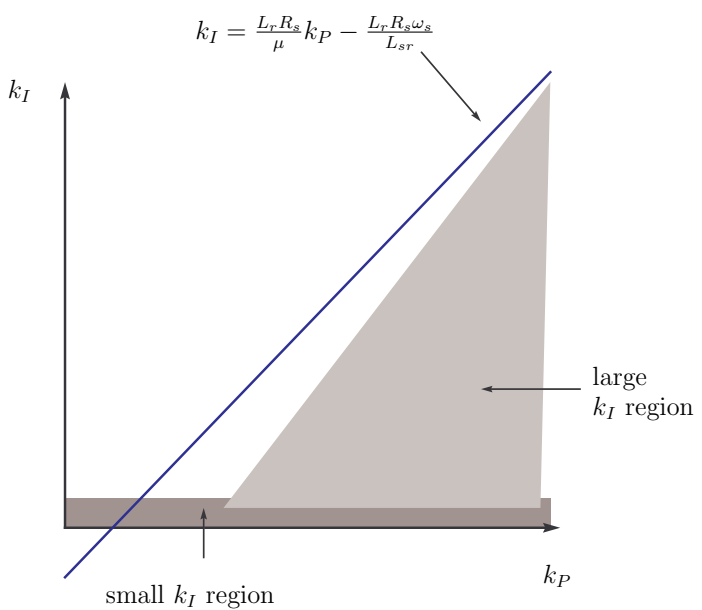

Fig. 2. Stability regions in the gains space. The height of the small $k_{I}$ region may actually vary with $k_{P}$.

propose to adopt the recent Immersion and Invariance (I\&I) technique proposed in [1]. The control is now replaced by

$$
v_{r}=\left(\omega_{s}-\omega\right) J_{2} \lambda_{r}+u+\left(\hat{R}_{r}+\beta\right) i_{r}
$$

where $\hat{R}_{r}$ is an estimate of $R_{r}$ and $\beta$ is a function to be defined that provides a new degree of freedom for the design. We define now the, so-called, off-the-manifold coordinate

$$
z=\hat{R}_{r}-R_{r}+\beta .
$$

It is easy to see that the new closed-loop system is

$$
\dot{\chi}=A \chi+B i_{r} z,
$$

where $\chi=\operatorname{col}\left(\tilde{i}_{s}, \tilde{i}_{r}, \int \tilde{i}_{s}\right)$ and

$$
\begin{gathered}
A=\left[\begin{array}{cc}
L^{-1} & O_{2} \\
O_{2} & I_{2}
\end{array}\right]\left[\begin{array}{ccc}
-\omega_{s} L_{s} J_{2}-R_{s} I_{2} & -\omega_{s} L_{s r} J_{2} & O_{2} \\
-k_{P} J_{2} & O_{2} & k_{I} J_{2} \\
I_{2} & O_{2} & O_{2}
\end{array}\right] \\
B=\frac{1}{\mu}\left[\begin{array}{c}
L_{s r} I_{2} \\
-L_{r} I_{2} \\
O_{2}
\end{array}\right]
\end{gathered}
$$

The objective in I\&I is not to cancel the uncertain term in a Lyapunov function derivative, like in classical adaptive control, but to generate an asymptotically stable dynamics for $z$. That is, we have to select $\hat{R}_{r}$ and $\beta$ so that $z(t) \rightarrow 0$. The result is summarized in the following proposition whose proof is given in the Appendix.

Proposition 2: Consider the system (1)-(3) in closed-loop with the control (5) and (9) with the adaptation laws

$$
\begin{gathered}
\beta=-\gamma \operatorname{sign}\left(i_{r d}\right) \lambda_{r d} \\
\dot{\hat{R}}_{r}=-\gamma\left|i_{r d}\right|\left(\hat{R}_{r}+\beta\right)+\gamma \operatorname{sign}\left(i_{r d}\right)\left[\left(\omega_{s}-\omega\right) \lambda_{r q}+v_{r d}\right],
\end{gathered}
$$

where $\gamma>0$ is an adaptation gain. Assume $i_{r d}$ is not absolutely integrable. Then, P1 and P2 of Proposition 1 hold true with the estimate $\hat{R}_{r}$ remaining bounded. 
The assumption that $i_{r d}$ is not absolutely integrable is essentially technical. As argued in the proof, roughly speaking, we only require that $\int\left|i_{r d}\right|$ be "sufficiently large". This will make $z$ "small enough" to be dominated by $A$. Furthermore, from the practical viewpoint, it can be shown that the situation $i_{r d}(t)=0$ in a compact time interval, is impossible in applications. Another observation pertains to our choice of the $d$-term of $i_{r}$. As indicated in the proof, it is possible to work also with the $q$-term, which choice is better will depend on the particular task that the DFIM is executing.

\section{Simulations}

In this section we implement a numerical simulation of the controller scheme developed in the previous sections. We use the following DFIM parameter values: $R_{s}=4.92 \Omega$, $R_{r}=4.42 \Omega, L_{s}=7.25 \mathrm{mH}, L_{r}=7.15 \mathrm{mH}, L_{s r}=7.1 \mathrm{mH}$, $J_{m}=0.00512 \mathrm{Kgm}^{2}, B_{r}=0.005 \mathrm{~N} \mathrm{~m} \mathrm{~s} \mathrm{rad}{ }^{-1}$.

As indicated in Section IV, the mechanical speed dynamics (3) can be stabilized by means of

$$
i_{s d}^{*}=\frac{1}{i_{r q}^{*}}\left(i_{s q}^{*} i_{r d}^{*}-\frac{B_{r}}{L_{s r}} \omega^{*}-\frac{\tau_{L}}{L_{s r}}+k_{\omega P} \tilde{\omega}+k_{\omega I} \int \tilde{\omega} d t\right)
$$

yielding the closed-loop behavior

$J \dot{\omega}=-B_{r}\left(\omega-\omega^{*}\right)-k_{\omega p}\left(\omega-\omega^{*}\right)-k_{\omega i} \int\left(\omega-\omega^{*}\right) d t+\epsilon_{t}$, where $\epsilon_{t} \rightarrow 0$ exponentially fast. Notice that the first three (constant) terms in (13) can be disregarded in a practical implementation, as their effect will be compensated by the integral part in any case.

The controller gains were fixed as $k_{P}=10, k_{I}=2$, $k_{\omega P}=1$ and $k_{\omega I}=25$. Simulations start with a desired mechanical speed $\omega^{*}=310 \mathrm{rad} \mathrm{s}^{-1}$ and at $t=0.5 \mathrm{~s}$ the desired value is changed to $\omega^{*}=325 \mathrm{rad} \mathrm{s}^{-1}$. The desired $q$-stator current is fixed at $i_{s q}^{*}=0$ in order to obtain a good power factor in the stator side.

Fig. 3 shows the behavior of the mechanical speed. The transient can be improved by means of the control gain of (13) and the integral term brings the mechanical speed to the desired value. Fig. 4 shows the behavior of $i_{s}$.

At $t=1.5 \mathrm{~s}$ the value of $R_{r}$ of the model is smoothly decreased to $R_{r}=3.42 \Omega$, simulating temperature effects. Fig. 5 show the estimation behavior of $R_{r}$. The convergence of $\hat{R}_{r}$ to the real value $R_{r}$ ensures that the performance of the ideal (known parameter) system is recovered.

\section{EXPERIMENTAL RESULTS}

For the experimental setup we used a $1.1 \mathrm{~kW}, 380 / 220 \mathrm{~V}$, $50 \mathrm{~Hz} 2$-poles machine, with the same parameters as Section VI. The three-phase rotor PWM voltages are generated by a bidirectional back-to-back converter [2]. The control algorithm is computed in a PC running with RTiC-Lab (Real Time Controls Lab) for Linux, with a $10 \mathrm{kHz}$ running time.

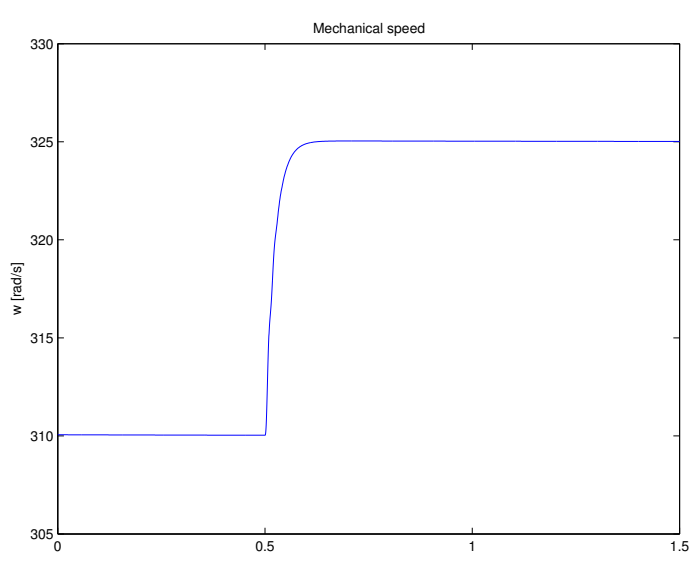

Fig. 3. Simulation results: mechanical speed, $\omega$.
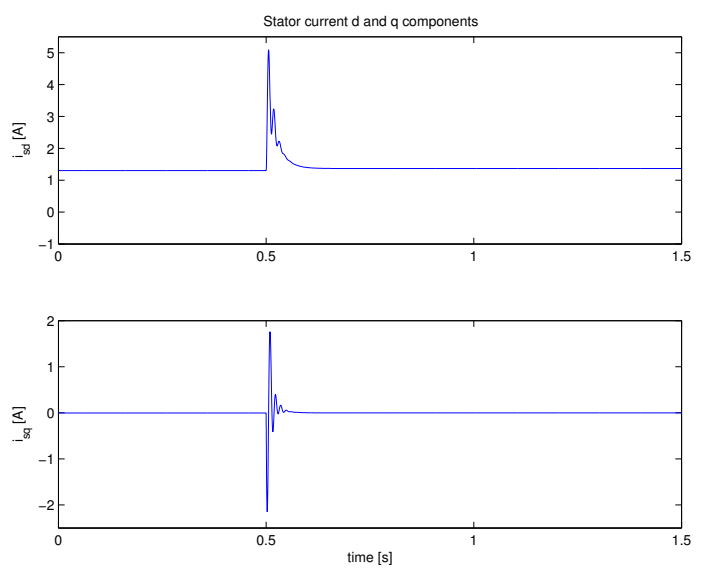

Fig. 4. Simulation results: stator current $d$ and $q$ components.

The experimental test consist of speeding up the machine from $\omega^{*}=310 \mathrm{rad} \mathrm{s}^{-1}$ to $\omega^{*}=325 \mathrm{rad} \mathrm{s}^{-1}$ and coming back to $\omega^{*}=310 \mathrm{rad} \mathrm{s}^{-1}$, and at the same time controlling the reactive power of the machine through $i_{s q}$.

In Fig. 6 the mechanical speed is depicted. Fig. 7 shows the dq-stator current components. Notice that $i_{s q}$ remains close to zero, which means that the power factor of the stator side is very small.

\section{CONCLUSIONS}

In this paper a particularly simple controller for DFIM was presented. It consists of a feedback linearizing term and a PI around stator currents. To improve the robustness of the feedback linearization stage an adaptive scheme that estimates the rotor resistance is also proposed. We prove that the scheme is globally asymptotically stable for all values of the proportional gain and sufficiently small integral gains. A region where large PI gains can be applied, preserving stability, is also identified. As no stator flux estimation is required, the algorithm scheme is simpler than the classical vector control. Simulations and experiments were used to 


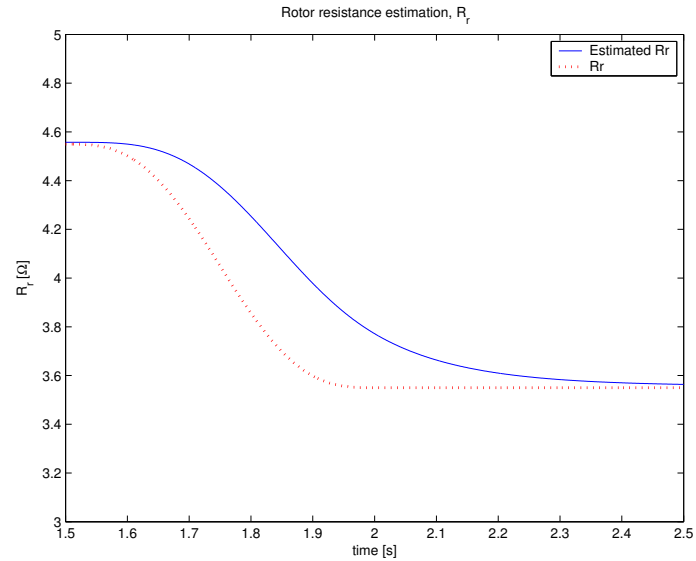

Fig. 5. Simulation results: convergence of the estimate $\hat{R}_{r}$.

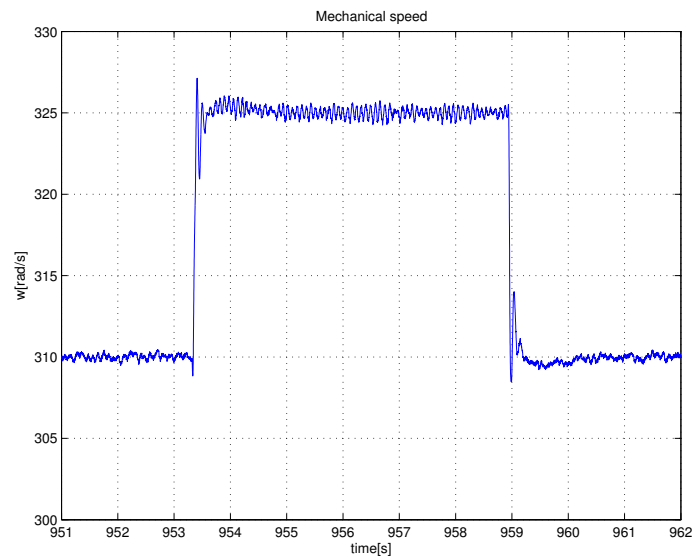

Fig. 6. Experimental results: mechanical speed $\omega$.

validate the control.

\section{REFERENCES}

[1] A. Astolfi and R. Ortega. Immersion and Invariance: A New Tool for Stabilization and Adaptive Control of Nonlinear Systems. IEEE Trans. Automat. Contr., 48(4):590-606, 2003.

[2] C. Batlle, A. Dòria-Cerezo, and E. Fossas. IDA-PBC controller for a bidirectional power flow full-bridge rectifier. In IEEE Proc. Conference on Decision and Control, pages 422-426, 2005.

[3] C. Batlle, A. Dòria-Cerezo, and R. Ortega. Power Flow Control of a Doubly-Fed Induction Machine Coupled to a Flywheel. European Journal of Control, 11(3):209-221, 2005.

[4] C. Batlle, A. Dòria-Cerezo, and R. Ortega. A Robustly Stable PI Controller for the Doubly-fed Induction Machine. In Proc. 32nd Annual Conference of the IEEE Industrial Electronics Society (IECON), 2006.

[5] M. Becherif, R. Ortega, E. Mendes, and S. Lee. Passivity-based control of a doubly-fed induction generator interconnected with an induction motor. In IEEE Proc. Conference on Decision and Control, 2003.

[6] P. Caratozzolo, E. Fossas, J. Pedra, and J. Riera. Dynamic modeling of an isolated motion system with DFIG. In Proc. IEEE International Power Electronics Congress, pages 287-292, 2000.

[7] A. Dòria-Cerezo. Modeling, simulation and control of a doubly-fed induction machine controlled by a back-to-back converter. $\mathrm{PhD}$ thesis, Universitat Politècnica de Catalunya, 2006.

[8] P. C. Krause. Analysis of electric machinery. McGraw-Hill, 1986.

[9] W. Leonard. Control of electric drives. Springer, 1995.
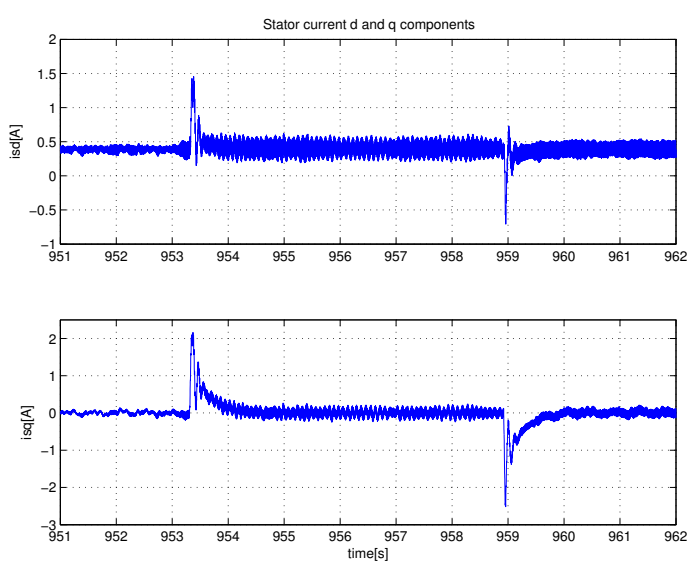

Fig. 7. Experimental results: stator current $d$ and $q$ components.

[10] R. Peña, J. C. Clare, and G. M. Asher. Doubly fed induction generator using back-to-back pwm converters and its application to variable speed wind-energy generation. In IEEE Proc. Electric Power Applications, volume 143-5, pages 231-241, 1996.

[11] S. Peresada, A. Tilli, and A. Tonelli. Power control of a doubly fed induction machine via output feedback. Control Engineering Practice, 12:41-57, 2004.

[12] W.J. Rugh. Linear system theory. Prentice Hall, 2nd edition, 1996.

[13] A. Tapia, G. Tapia, J. X. Ostolaza, and J. R. Sáenz. Modeling and control of a wind turbine driven doubly fed induction generator. IEEE Trans. Energy Conversion, 18:194-204, 2003.

\section{APPENDIX}

Proof of Proposition 2. First, we note that the $d$ equation of (2) can be written as

$$
\dot{\lambda}_{r d}=\left(\omega_{s}-\omega\right) \lambda_{r q}+v_{r d}-R_{r} i_{r d} .
$$

Replacing this expression in (12) yields

$$
\dot{\hat{R}}_{r}=-\gamma\left|i_{r d}\right|\left(\hat{R}_{r}+\beta\right)+\gamma \operatorname{sign}\left(i_{r d}\right)\left(\dot{\lambda}_{r d}+R_{r} i_{r d}\right) .
$$

On the other hand, differentiating (11) one gets

$$
\dot{\beta}=-\gamma \operatorname{sign}\left(i_{r d}\right) \dot{\lambda}_{r d} .
$$

Replacing these two terms in $\dot{z}=\dot{\hat{R}}_{r}+\dot{\beta}$ yields

$$
\dot{z}=-\gamma\left|i_{r d}\right| z .
$$

The solution of this differential equations is

$$
z(t)=e^{-\gamma \int_{0}^{t}\left|i_{r d}(\tau)\right| d \tau} z(0) .
$$

Since $i_{r d}$ is not absolutely integrable $z(t) \rightarrow 0$ as $t \rightarrow \infty$.

To complete the proof we rewrite the closed loop system (10) in the form

$$
\dot{\chi}=(A+B D z(t)) \chi+B E z(t),
$$

where we have used the fact that $i_{r}=D \chi+E$, for some constant matrix $D$ and constant vector $E$. This is a linear time-varying system that asymptotically converges to the linear time-invariant asymptotically stable system $\dot{\chi}=A \chi$. Hence, invoking standard arguments, e.g., Exercise 8.7 of [12], we conclude that $x(t) \rightarrow 0$. 